\title{
SMRT sequencing of the full-length transcriptome of the Rhynchophorus ferrugineus (Coleoptera: Curculionidae)
}

\author{
Hongjun Yang ${ }^{1}$, Danping Xu ${ }^{2}$, Zhihang Zhuo ${ }^{\text {Corresp.. } 1,3}{ }^{\text {, Jiameng Hu }}{ }^{1}$, Baoqian $\mathrm{Lu}^{4}$ \\ ${ }^{1}$ Key Laboratory of Genetics and Germplasm Innovation of Tropical Special Forest Trees and Ornamental Plants, Ministry of Education, Key Laboratory of \\ Germplasm Resources Biology of Tropical Special Ornamental Plants of Hainan Province, College of Forestry, Hainan University, Haikou, Hainan, China \\ 2 Sichuan Provincial Key Laboratory of Agricultural Products Processing and Preservative, College of Food Science, Sichuan Agricultural University, Yaan, \\ Sichuan, China \\ 3 \\ ${ }^{3}$ Key Laboratory of Integrated Pest Management on Crops in South China, Ministry of Agriculture, South China Agricultural University, Guangzhou, \\ Guangdong, China \\ ${ }^{4}$ Key Laboratory of Integrated Pest Management on Tropical Crops, Ministry of Agriculture China, Environment and Plant Protection Institute, Chinese \\ Academy of Tropical Agricultural Sciences, Haikou, Hainan, China \\ Corresponding Author: Zhihang Zhuo \\ Email address: zhuozhihang@foxmail.com
}

Background. Red palm weevil Rhynchophorus ferrugineus (Coleoptera: Curculionidae) is one of the most destructive insects for palm trees in the world. However, its genome resources are still in the blank stage, which limits the study of molecular and growth development analysis. Methods. In this study, we used PacBio Iso-Seq and Illumina RNAseq to first generate transcriptome from three developmental stages of $R$. ferrugineus (pupa, 7th larva, female and male) to increase our understanding of the life cycle and molecular characteristics of $R$. ferrugineus. Results. A total of 63,801 nonredundant fulllength transcripts were generated with an average length of 2,964 bp from three developmental stages, including the 7th instar larva, pupa, female adult and male adult. These transcripts showed a high annotation rate in seven public databases, with 54,999 (86.20\%) successfully annotated. Meanwhile, 2,184 alternative splicing (AS) events, 2,084 transcription factors (TFs), 66,230 simple sequence repeats (SSR) and 9,618 Long noncoding RNAs (IncRNAs) were identified. In summary, our results provide a new source of full-length transcriptional data and information for the further study of gene expression and genetics in $R$. ferrugineus. 
1 SMRT sequencing of the full-length transcriptome of

2 the Rhynchophorus ferrugineus

3 (Coleoptera:Curculionidae)

4

Hongjun Yang ${ }^{1}$, Danping $\mathrm{Xu}^{2}$, Zhihang Zhuo ${ }^{1,3, *}$, Jiameng $\mathrm{Hu}^{1}$, Baoqian $\mathrm{Lu}^{4}$

${ }^{1}$ Key Laboratory of Genetics and Germplasm Innovation of Tropical Special Forest Trees and

8 Ornamental Plants, Ministry of Education, Key Laboratory of Germplasm Resources Biology of

9 Tropical Special Ornamental Plants of Hainan Province, College of Forestry, Hainan University, 10 Haikou, China

$11{ }^{2}$ Sichuan Provincial Key Laboratory of Agricultural Products Processing and Preservative, 12 College of Food Science, Sichuan Agricultural University, Yaan, Sichuan, China

$13{ }^{3}$ Key Laboratory of Integrated Pest Management on Crops in South China, Ministry of 14 Agriculture, South China Agricultural University, Guangzhou, Guangdong, China

$15{ }^{4}$ Key Laboratory of Integrated Pest Management on Tropical Crops, Ministry of Agriculture

16 China, Environment and Plant Protection Institute, Chinese Academy of Tropical Agricultural

17 Sciences, Haikou, Hainan, China

18

19

20

21

22

23

24

25

26

27

28

29

30

31

32

33

34

35

36

37

38

39

40

\section{Corresponding Author:}

Zhihang Zhuo ${ }^{1}$

No. 58 Renmin Avenue, Haikou City, Hainan Province, 570228, China

Email address: zhuozhihang@foxmail.com 
41 Abstract

42 Background. Red palm weevil Rhynchophorus ferrugineus (Coleoptera: Curculionidae) is one

43 of the most destructive insects for palm trees in the world. However, its genome resources are

44 still in the blank stage, which limits the study of molecular and growth development analysis.

45 Methods. In this study, we used PacBio Iso-Seq and Illumina RNA-seq to first generate

46 transcriptome from three developmental stages of $R$. ferrugineus (pupa, 7th larva, female and

47 male) to increase our understanding of the life cycle and molecular characteristics of $R$.

48 ferrugineus.

49 Results. A total of 63,801 nonredundant full-length transcripts were generated with an average

50 length of $2,964 \mathrm{bp}$ from three developmental stages, including the 7 th instar larva, pupa, female

51 adult and male adult. These transcripts showed a high annotation rate in seven public databases,

52 with 54,999 (86.20\%) successfully annotated. Meanwhile, 2,184 alternative splicing (AS) events,

532,084 transcription factors (TFs), 66,230 simple sequence repeats (SSR) and 9,618 Long

54 noncoding RNAs (lncRNAs) were identified. In summary, our results provide a new source of

55 full-length transcriptional data and information for the further study of gene expression and

56 genetics in R. ferrugineus.

57 Introduction

58 The red palm weevil (RPW), Rhynchophorus ferrugineus (Coleoptera: Curculionidae), is the world's most notorious pest that destroys palm trees (Vatanparast et al., 2014; Wakil, Faleiro \& Miller, 2015). It originated in Southeast Asia and rapidly spread to the rest of the world, including the entire Mediterranean region, Asia and Oceania, destroying the coastal palm trees and threatening the production of coconut trees (Soroker et al., 2005). In China, $R$. ferrugineus is an alien invasive pest. Since its invasion, $R$. ferrugineus has led to the death of about 20,000 coconut trees, with an area of more than 10,000 square kilometers, which seriously threatens the ecological security of coastal areas (Shi, Lin \& Hou, 2014; Ge et al., 2015). As larva extensively feeds on tissues, the pest would cause extensive damage to the plant's apical meristem, threaten the survival of plants and impair the mechanical stability of plants. When red palm weevil damages on palm trees, the main symptoms are only visible in the later stages of infestation. At this time, the larva has already destroyed the apical meristem, and any control measures are ineffective, eventually causing the infected palm to collapse and dump (Ferry \& Gomez, 2002; Sacchetti, 2006; Hussain et al., 2013).The biological properties and continuous feeding behavior of $R$. ferrugineus make it impossible to effectively control this pest by all conventional methods, especially chemical pesticides. Research on biological control strategies, mainly microbes, provides a new approach for controlling $R$. ferrugineus, but their application remains a relatively long-term goal (Blumberg, 2008; Mazza et al., 2014). Transcriptomes data reflects the information of cellular responses, gene function, evolution and reveal different biological processes at the molecular level (Hittinger et al., 2010; Jia et al., 2018). Transcriptome analysis improves the understanding of molecular responses and hopefully provide evidence for the 
80

81

82

83

84

85

86

87

88

89

90

91

92

93

94

95

96

97

98

99

100

101

102

103

104

105

106

107

108

109

110

111

112

113

114

115

116

117

118

119

research of transcriptome mainly focuses on insect resistance, feeding behavior, mating behavior and oviposition, which will provide new ideas to further study of red palm weevil.

Short-reading transcriptome sequencing has been widely used to describe gene expression levels, and many model organisms and non-model organism transcriptomes were obtained by short-sequence sequencing on a second-generation sequencing platform (Nagalakshmi et al., 2008; Ekblom \& Galindo, 2011; Djebali et al., 2012). Recently, the expression analysis of Coleoptera insects at different developmental stages have been studied using the next-generation sequencing method (Won et al., 2018; Chanchay et al., 2019; Noriega et al., 2019). Additionally, the research on transcriptome of $R$. ferrugineus intestinal microbes showed those microbes in the intestine of larva had profound effects on the immune stimulation and nutritional metabolism (Habineza et al., 2019; Muhammad et al., 2019). However, the spliced transcripts of short-reading transcriptome sequencing are incomplete, and the current short sequence sequencing prediction program is difficult to accurately predict the gene structure (Coghlan et al., 2008). Furthermore, low-quality transcripts obtained by short-sequencing sequencing may result in incorrect annotations (Lin et al., 2017; Li et al., 2018). The second-generation sequencing technology has defect of short read length and can not span the entire transcripts (Koren et al., 2012). Nevertheless, third-generation long-read sequencing platforms can overcome those difficulties.

Compared with short-reading transcriptome sequencing, full-length transcriptome sequencing is based on the PacBio Sequel third-generation sequencing platform, which will directly obtain complete transcripts containing 5'UTR, $3^{\prime} \mathrm{UTR}$, and polyA tails without interrupting splicing, thereby accurately analyzing reference genomic species. The study on structural information of full-length transcriptome sequencing, such as alternative splicing and fusion genes, solves the problem of short splicing and incomplete information of transcripts without reference genomes. Currently, single molecule real-time long read sequencing (SMRT) is one of the most reliable full-length cDNA molecular sequencing methods. It has been successfully applied to the fulllength transcriptome analysis of human, animals, plants and insects to obtain more authentic transcriptome information reflecting intact species sequence (Sharon et al., 2013; Larsen, Campbell \& Yoder, 2014; Abdel-Ghany et al., 2016; Hartley et al., 2016; Wang et al., 2016; Chen et al., 2017; Zhu et al., 2017; Kawamoto et al., 2019). To the best of our knowledge, there are few reports on full-length transcriptome sequencing of $R$. ferrugineus at present, especially for gene expression analysis at different developmental stages.

In this work, methods of short-reading transcriptome sequencing (Illumina RNA-seq) combined with full-length transcriptome sequencing (PaBio Iso-seq) were applied to obtain a complete full-length transcriptome of $R$. ferrugineus which would be beneficial to comprehensively analyze its transcriptome information. Then, functional annotation, CDS (Coding sequence) prediction, simple sequence repeats analysis, and transcription factors analysis were performed on the complete full-length transcriptome. Finally, lncRNAs and alternative splicing events were analyzed. Here, we performed full transcriptome sequencing for species without a reference genome, providing a complete set of genome reference 
120 (transcriptome sequences) of $R$. ferrugineus, supplying a reference for further analysis of gene

121 expression profile, and exhibiting valuable resources for future molecular biology research of red

122 palm weevil.

\section{Materials \& Methods}

124 Samples selection and preparation

125 All R. ferrugineus samples used in this study were collected from the Coconut Research Institute, 126 Chinese Academy of Tropical Agricultural Sciences, Wenchang Hainan, China. Samples were 127 divided into larva, pupa, female adult and male adult. The whole body was collected from $12 R$. 128 ferrugineuss ( 3 from 7th instar larvae, 3 from Pupae, 3 from female adults, 3 form male adults). 129 All samples were harvested and frozen in liquid nitrogen and stored at $-80^{\circ} \mathrm{C}$ for further 130 experiments.

131 RNA extraction and SMRT sequencing

132 Total RNA samples were isolated using the RNeasy Plus Mini Kit (Qiagen, Valencia, CA, USA). 133 Then 1\% agarose gels was used to detect RNA degradation and contamination, and Nanodrop 134 (NanoDrop products, USA) was used to check RNA purity (OD 260/280). RNA concentration 135 and integrity were accurately evaluated using Qubit ${ }^{\circledR}$ RNA Assay Kit in Qubit ${ }^{\circledR} 2.0$ 136 Flurometer (Life Technologies, CA, USA) and Agilent 2100 (Agilent Technologies, USA), 137 respectively. For PacBio Iso-Seq, the total RNA samples from three developmental stages (larva, 138 Pupa, female adult and male adult) were mixed together for the following experiments. A total of $1393 \mu \mathrm{g}$ of mixed RNA was sequenced on the Pacbio Sequel platform (Pacific Biosciences, CA, 140 USA) in accordance with the manufacturer's instructions. Then, according to the Isoform 141 sequencing protocol (Iso-Seq), the Iso-Seq library was prepared by the Clontech SMARTer PCR 142 cDNA synthesis kit (Clontech, CA, USA) and the BluePippin size selection system protocol 143 described by Pacific Biosciences (PN 100-092-800-03). For Illumina RNA-Seq, twelve libraries 144 of three developmental stages (larva, pupa, male adult and female adult) RNA samples were 145 prepared and sequenced respectively. For each sample, a total amount of $1.5 \mu \mathrm{g}$ RNA was used 146 for short reads sequencing on Novaseq 6000 platform. Sequencing libraries were generated using 147 NEBNext ${ }^{\circledR}$ Ultra ${ }^{\mathrm{TM}}$ RNA Library Prep Kit for Illumina ${ }^{\circledR}$ (NEB, USA) following 148 manufacturer's recommendations and index codes were added to attribute sequences to each 149 sample. The sequencing work reported in this work was performed by Novogene technology co. 150 (Beijing, China). PacBio Iso-Seq and Illumina RNA-seq data generated from $R$. ferrugineus are 151 available from the NCBI SRA database under project number PRJNA598560.

152 Data processing and error correction of PacBio Iso-Seq reads

153 Firstly, SMRTlink 6.0 software was used to process the sequence data. Immediately after, the 154 cyclic consensus sequence (CCS) was generated from the subread BAM files (parameters: 155 min_length 50, max_drop_fraction 0.8, no_polish TRUE, min_zscore-9999.0, min_passes2, 156 min_predicted_accuracy 0.8, max_length 15000) and a CCS.BAM file was output. The 157 generated BAM files were divided into full-length and non-full-length reads using pbclassify. 158 Finally, input the full and non-full length fasta files into the clustering step, which performs the 159 isoform level clustering, and then uses Quiver (parameters: hq_quiver_min_accuracy 0.99, 
160 bin_by_primer false, bin_size_kb 1, qv_trim_5p 100, qv_trim_3p 30) for the final arrow

161

162

163

164

165

166

167

168

169

170

171

172

173

174

175

176

177

178

179

180

181

182

183

184

185

186

187

188

189

190

191

192

193

194

195

196

197

198

199

polishing. Full-length transcripts were corrected using Illumina RNA-seq data with the software LoRDEC (Salmela \& Rivals, 2014). The redundancies in the corrected transcript were then removed using the CD-HIT-EST (parameters: -c 0.95 -T 6 -G 0 -aL 0.00 -aS 0.99) program to obtain the final transcript for subsequent analysis (Fu et al., 2012).

\section{Functional annotation of transcripts}

Transcripts function was annotated based on the following databases:NR (NCBI non-redundant protein sequences) (Deng et al., 2006), NT (NCBI non-redundant nucleotide sequences), Pfam (Protein family) (Finn et al., 2014), KOG (Clusters of Orthologous Groups of proteins) (Koonin et al., 2004), Swiss-Prot (A manually annotated and reviewed protein sequence database) (Apweiler et al., 2004), KEGG (Kyoto Encyclopedia of Genes and Genomes) (Kanehisa et al., 2004) and GO (Gene Ontology) (Ashburner M, 2000). The BLAST software with E-value $\leq 1 \mathrm{e}-5$ was used for NT database analysis. The Diamond v0.8.36 software with E-value $\leq 1 \mathrm{e}-5$ was analyzed in NR, KOG, Swiss-Prot and KEGG annotations. The Hmmscan procedure was used in the Pfam database, and GO function categories were performed by Blast2GO v2.5 based on Pfam annotation.

\section{CDS prediction and SSR analysis}

The ANGEL pipeline is a long-read implementation of ANGLE that is used to determine protein coding sequences from cDNAs. We use the confidence protein sequences of $R$. ferrugineus or closely related species for ANGLE training, and then run the ANGLE predictions for given sequences (Shimizu, Adachi \& Muraoka, 2006). At the same time, the MISR (http://pgrc.ipkgatersleben.de/misa/misa.html) was used to identify and locate the SSR of the transcriptome.

\section{Identification of TFs and IncRNAs}

Animal transcription factors were performed by the animal TFDB 2.0 database (Zhang et al., 2015). For species included in the database, if they were not Ensembl geneid genes, they would be screened by BLASTX with the known transcription factors protein sequence of the species in the database, and if they were Ensembl geneid, they would be screened directly. For species not included in the database, hmmsearch was used to identify them according to pfam files of the transcription factor family. LncRNA of the transcriptome were predicted by using Coding-NonCoding-Index (CNCI) (Altschul et al., 1997), Coding Potential Calculator (CPC) (Kong et al., 2007), Pfam-scan (Finn et al., 2016) and PLEK (Li, Zhang \& Zhou, 2014) to predict the coding potential of transcripts. Firstly, PLEK SVM classifier with default parameters of -minlength 200 and CNCI with default settings were performed to evaluate coding potential; Secondly, CPC and NCBI eukaryotic protein database were used for BLAST comparison ( E-value $<1 \mathrm{e}-10$ setting) to search transcripts; Finally, homologous search of hmmscan was performed for the transcription sequences predicted by the three software with the Pfam database, and the protein family domains were recorded, with the default parameter of -E 0.001-domE 0.001 .

\section{AS Analysis}

To obtain alternative splicing (AS) events for R. ferrugineus, transcripts were further processed using Coding GENome reconstruction tool (Cogent v3.1, https://github.com/Magdoll/Cogent). 
200 Generally, Cogent first divides the input fasta file into chunk_size blocks, and then calculates the 201 k-mer configuration file. Then, the De Bruijn graph was used to further reconstruct each 202 transcription family into one or more unique transcription models (called UniTransModels). 203 Finally, gmap-2017-06-20 was conducted to map the adjusted transcripts to UniTransModels.

204 Splicing junctions detection was performed on transcripts mapped to the same UniTransModels, 205 and transcripts with the same splice junctions were collapsed. Meanwhile, the transcriptional 206 isoforms of UniTransModels have collapsed transcripts with different splicing junctions. AS 207 events were detected with SUPPA (https://github.com/comprna/SUPPA) using default settings.

\section{Results}

209 The full-length sequences of $\boldsymbol{R}$. ferrugineus using PacBio sequencing

210 The full-length transcriptome of $R$. ferrugineus was generated using the PacBio Sequel platform 211 on the pooled RNA of twelve R. ferrugineus samples. The results showed that PacBio Sequel 212 platform generated a total of 454,369 circular consensus sequences (CCSs) with a full length 213 reads of 362,466. The nonfull-length (nFL) sequences was 81,424 and the full-length non 214 chimera (FLNC) reads number was 330,973 with an average length of $2,332 \mathrm{bp}$. Twelve samples 215 were sequenced by Illumina Novaseq 6000 respectively, and a total of 642,179,304 raw reads 216 and 625,983,256 clean reads $(97.48 \%, 93.91 \mathrm{G})$ were obtained. PacBio Sequel platform 217 produced a total of $10,172,136$ subreads and 181,405 consensus reads $(16.67 \mathrm{G}$, with an average 218 length of 2,305 bp, an N90 of 1,327 bp and an N50 of 2,790 bp), which were then corrected 219 using the Illumina reads (after correction, the average length was 2,302 bp, N90 was 1,321 bp, 220 221

222

223

224

225

226

227

228

229

230

231

232

233

234

235

236

237

238

239 and N50 was 2,785 bp) and ubsequently removing redundancy via the CD-Hit program, the consensus transcripts were finally clustered into a total of 63,801 transcripts for subsequent analysis. We found that the main length distribution range of unigenes was 0.5 -6k (Fig. 1).

\section{Gene annotation of $R$. ferrugineus}

To obtain a comprehensive functional annotation of R. ferrugineus transcriptome, we annotated 63,801 transcripts with seven databases, including Swiss-Prot, KOG, GO, NR, NT, Pfam, and KEGG. In total, 50,280, 40,109, 47,197, 33,511, 27,707, 27,253 and 27,707 transcripts were annotated in the NR, Swiss-Prot, KEGG, KOG, GO, NT and Pfam databases, respectively. Moreover, 54,999 transcripts were annotated in at least one database and 12,508 transcripts were annotated in all databases (Fig. 2).

NR is a non-redundant protein database characterized by its comprehensive content and the inclusion of species information in the annotated results, which can be used for the classification of homologous species. Aligned all transcripts in the NR database, the results showed that 50,280 transcripts were annotated in the NR database and the top five most annotated in NR database were Dendroctonus ponderosae, Anoplophora glabripennis, Bactrocera tryoni, Tribolium castaneum and Aethina tumida (Fig. 3).

KOG is a protein database created and maintained by NCBI, which is constructed according to the phylogenetic relationship of coding proteins in complete genomes of bacteria, algae and eukaryotes, and is widely used to predict the function of sequences. The KOG functional classification of $R$. ferrugineus transcripts was shown in Fig. 4. The results indicated that a total 
240

241

242

243

244

245

246

247

248

249

250

251

252

253

254

255

256

257

258

259

260

261

262

263

264

265

266

267

268

269

270

271

272

273

274

275

276

277

278

279

of 33,511 genes categorizing into 26 categories were annotated in KOG database. The first six largest groups among these categories were transcription, general function prediction only, function unknown, signal transduction mechanisms, cytoskeleton and posttranslational modification (protein turnover and chaperones), respectively.

Full-length transcripts of red palm weevil were annotated with GO database, and 27,707 annotated transcripts were successfully divided into three categories: biological processes, molecular functions, and cellular Components (Fig. 5). In biological process, the cell process accounts for the largest proportion, followed by metabolic process and single-organism process. In addition, we also found that part of the genes was annotated into biological regulation, regulation of biological process, localization, response to stimulus and signaling terms. In cellular component, the genes involved in cell, cell part, organelle, membrane, membrane part and macromolecular complex were the most. In molecular function categories, binding, catalytic activity and transporter activity were identified as the most abundant terms.

KEGG Pathway analysis can be used to systematically analyze the metabolic pathways of gene products and compounds in cells and the functions of these gene products. In the KEGG classification of $R$. ferrugineus, human diseases, metabolism and organismal systems were the top three categories with higher proportions (Fig. 6). Briefly, a total of 11,950 genes were involved in human disease related pathways, in which 1,668 genes were predicted to infectious disease: viral, 1,396 genes were predicted tonfectious diseases: bacterial and 2,777 genes were predicted to cancers: overview. A total of 10,294 of the annotated genes were classified as belonging to organismal systems related pathways, in which the nervous system (1,016 genes), immune system (1,984 genes), digestive system (993 genes) and endocrine system (2,500 genes) were the top four pathways with the most abundant genes. In addition, a total of 7,979 annotated genes were involved in the Metabolism pathway. The most abundant pathways were carbohydrate metabolism (1,478 genes) and amino acid metabolism (1,180 genes). Regarding Environmental Information processing, genes were involved in the signal transduction $(4,741$ genes), signaling molecules and interaction (304 genes) and membrane transport (196 genes). At the same time, a lower number of genes are annotated to Cellular Processes and Genetic Information Processing.

\section{CDS prediction}

The CDS is a sequence encoding a protein product that corresponds exactly to the codon of a protein. In the sequencing results of the full-length transcriptome, predicting the protein coding region contributes to the preliminary analysis of the gene and is also the basis for subsequent protein structure analysis. For red palm weevil, ANGEL software was performed to carry out CDS prediction analysis on the obtained full-length transcriptome sequence, and the results showed that the main distribution range of CDS length was 0 2500nt (Fig. 7).

\section{Transcription factors identification}

Transcription factors are an important part of the transcriptional regulatory system. Using the present data of $R$. ferrugineus, 2,084 transcription factors were predicted, and Zf-C2H2 (570,27.35\%), ZBTB (476,22.84\%), TF_bzip (101,4.85\%) and bHLH $(85,4.08 \%)$ were the top 
280 four transcription factor families (Fig. 8). These transcription factors will lay the foundation for 281 exploring the role of the regulatory mechanism of red palm weevil.

\section{SSR discovery}

283 Simple sequence repeats is a group of repeated sequences consisting of several nucleotides (1 6)

284 with repeat units up to dozens of nucleotides. The repeats are short in length and widely

285 distributed uniformly in eukaryotic genomes. In our analysis, MISA software (version 1.0,

286 default parameter) was applied for SSR detection of transcriptome. The results showed that the

287 minimum number of repetitions of each unit size is 1-10, 2-6, 3-5, 4-5, 5-5, 6-5. In total 66,230

288 SSR loci were identified in this transcriptome; mono nucleotide motifs $(49,898,75.34 \%)$ were

289 the most abundant type of SSR locus, followed by di nucleotide motifs $(12,662,19.12 \%)$, tri

290 nucleotides $(3,377,5.09 \%)$, tetra nucleotides $(192,0.29 \%)$, penta nucleotides $(33,0.05 \%)$ and

291 hexa-nucleotide motifs (68, 0.10\%) (Fig. 9).

\section{LncRNA prediction}

293 LncRNA (long-chain noncoding RNA) is a class of RNA molecules whose transcripts are more 294 than $200 \mathrm{nt}$ in length and do not encode proteins. For $R$. ferrugineus, the numbers of lncRNAs 295 identified from transcriptome by CNCI, CPC, PLEK and Pfam were 32,552, 17,481, 18,897 and 296 34,066, respectively (Fig. 10). The intersection of these four results produced 9,618 lncRNA 297 transcripts. Meanwhile, the length distribution density of mRNA was compared with the 298 predicted lncRNA (Fig. 11).

\section{Alternative splicing analysis from full-length transcriptomes}

300 Since $R$. ferrugineus had no reference genome, we used Cogent (Coding genome reconstruction 301 tool) to reconstruct genes using high-quality full-length transcriptome sequences to generate 302 UniTransModels. UniTransModels were used as a reference sequence to describe the types of 303 AS events and the number of corresponding genes. The results indicated that a total of 2,184 304 UniTransModels-based AS events in R. ferrugineus were detected. Briefly, six main AS events 305 (alternative 3' splice sites, Mutually exclusive exons, Skipping Exon, alternative 5' splice 306 sites, Retained introns and Alternative First Exons) were identified. Retained introns (RI) were 307 identified as the most abundant event, accounting for 6.14\% (134) of all events. The other five 308 types of AS events account for less than $2 \%$ of all detectable events. The number of two kinds of 309 events, alternative $3^{\prime} \quad$ splice sites $(32,1.47 \%)$ and alternative $5^{\prime} \quad$ splice sites $(34,1.56 \%)$, were 310 slightly higher than those of skipped exons (7,0.32\%), mutually exclusive exons $(1,0.05 \%)$ and 311 Alternative first exons (20,0.92\%).

\section{Discussion}

313 In recent years, with the development of sequencing technology, transcriptome sequencing has

314 become an important mean to study the regulation of gene expression. The third-generation 315 sequencing (full-length transcriptome sequencing) captures full-length transcripts without 316 assembly, overcoming the difficulties of the second-generation sequencing (short-reading 317 transcriptome sequencing). Besides, the third-generation sequencing contributes to the following 318 fields: accurately reflect the transcriptome information of the sequenced species; detect multiple 319 variable splicing forms, and find more splicing sites and alternative splicing events; find new 
320 functional genes, and supplement the genome annotation; accurately analyze fusion genes,

321

322

323

324

325

326

327

328

329

330

331

332

333

334

335

336

337

338

339

340

341

342

343

344

345

346

347

348

349

350

351

352

353

354

355

356

357

358

359

homologous genes, superfamily genes and alleles. According to the report, for Oxya chinensis, Acrida cinerea and Atractomorpha sinensis, the total length, average length, N50 and N90 of transcripts obtained by PacBio RS II platform (full-length transcriptome) all greater than those obtained by RNA-seq transcriptomes (short-reading transcriptome) (Zhao, 2018). Although the third-generation sequencing technology represented by PacBio Iso-Seq has the superiority of extremely long reading length, its reading error rate of single-base is pretty high (up to 15\%), which can be corrected by second-generation short reads (Au et al., 2013; Li et al., 2014). In this work, we pooled and sequenced RNA samples from different developmental stages of $R$. ferrugineus, using both PacBio Iso-Seq and Illumina RNA-seq to obtain full-length transcriptome data, providing a general encyclopedia of gene transcription. As expected, massive transcriptome data of $R$. ferrugineus was generated, including 63,801 full-length transcripts with $2,964 \mathrm{bp}$ of average length and 3,547 bp of N50 length. The amount of transcriptome data acquired in red palm weevil was much higher than that in Coleoptera insects with different developmental stages by second-generation sequencing, such as Hypothenemus hampei (average length 1,609.92 bp and N50 length 2,427 bp ) (Noriega et al., 2019), Nicrophorus orbicollis (average length 1,193 bp and N50 length 2,856 bp) (Won et al., 2018), Sclerotia aquatilis (average length 1,394 bp and N50 length 2,666 bp) (Chanchay et al., 2019), and Batocera horsfieldi (average length 1,188 bp and N50 length 3,143 bp) (Yang et al., 2018). Meanwhile, a total of 54,999 (86.20\%) among 63,801 transcripts of $R$. ferrugineus were successfully annotated as known homologous genes using seven databases.

In the study of gene annotation, a large number of new transcripts can be classified for obtaining gene function information. The updated collection of homologous protein sets of prokaryotes and eukaryotes is expected to be used for functional annotation of newly sequenced genomes, including those complex eukaryotes, as well as genome-wide evolutionary studies (Tatusov et al., 2003). Genomic sequencing has made it clear that a large fraction of the genes specifying the core biological functions are shared by all eukaryotes (Ashburner et al., 2000). In SMRT sequencing of the full-length transcriptome of the $R$. ferrugineus, a total of $27,707 \mathrm{FL}$ transcripts were annotated into the GO database, most of which were biological processes, followed by cellular components and molecular functions. A total of 47,197 transcripts of red palm weevil were annotated to $40 \mathrm{KEGG}$ pathways, the top four most annotated KEGG pathways were signal transduction, cancers (specific types), transport and catabolism, endocrine system. In addition, the KOG annotation results showed that the transcripts associated with General function prediction only and Signal transduction mechanisms were the most. The results of gene annotation indicated that the new transcripts were related to the above functions.

AS events have attracted the attention of biologists as an important mechanism to increase protein diversity and regulate gene expression (Thatcher et al., 2016; Vuong, Black \& Zheng, 2016). AS occurs by rearranging the pattern of intron and exon elements that are joined by splicing to alter the mRNA coding sequence (Braun et al., 2018). PacBio long-read transcriptome sequencing is superior to short-read RNA-Seq in the recognition of AS events

Peer) reviewing PDF | (2019:12:44425:1:1:NEW 20 Mar 2020) 
360

361

362

363

364

365

366

367

368

369

370

371

372

373

374

375

376

377

378

379

380

381

382

383

384

385

386

387

388

389

390

391

392

393

394

395

396

397

398

399

(Tilgner et al., 2014; Weirather et al., 2015). At the same time, the accuracy of the PacBio transcript to identify AS events has been verified (Abdel-Ghany et al., 2016; Wang et al., 2016). In our work, third-generation sequencing technology was adopted to capture 2,184 AS events from the FL transcripts.

lncRNAs mainly regulate gene expression at the epigenetic level through transcriptional regulation and post-transcriptional regulation, and exert powerful biological functions by affecting protein localization and telomere replication (Batista \& Chang, 2013; Kung, Colognori \& Lee, 2013; Qureshi \& Mehler, 2013). In recent years, a large number of IncRNAs have been identified from insects, such as Apis mellifera, Nasonia vitripennis and Nilaparvata lugens, which laid an important foundation for further study of the function of lncRNAs in insect growth and development(Zhu, Liang \& Gao, 2016). Furthermore, the functions of lncRNAs in Drosophila have been extensively studied in multiple insect species. For example, lncRNA can be involved in regulating the sex determination process of Drosophila (Mulvey et al., 2014), male courtship behavior (Chen et al., 2011), motor behavior and climbing ability (Li et al., 2012), inactivation of X chromosome (Smith, Allis \& Lucchesi, 2001), and sleep behavior (Soshnev et al., 2011). In Plutella xylostella, the lncRNA regulates the resistance of the insect to bacillus thuringiensis (bt) endotoxin Cry1 Ac, phenylpyrazole and chlorpyrifos (Etebari, Furlong $\&$ Asgari, 2015). In addition to regulating growth and drug resistance, lncRNAs have a rapid response to stress and stimulation (Lakhotia, 2012; Mizutani et al., 2012; Valluri, Rupam \& Srividya, 2017; Li et al., 2019). Many studies have confirmed that lncRNAs can modulate multiple immune responses, including several pathways related to innate immunity (Fitzgerald \& Caffrey, 2014; Heward \& Lindsay, 2014; Ahmed \& Liu, 2018). Simultaneously, some transcription factors may be involved in different metabolic processes and may have multiple different functions (Chen \& Rajewsky, 2007). For example, GATA and FoxA transcription factors play an important role in the differentiation and maintenance of different tissues by controlling gene expression (Boyle \& Seaver, 2010; Zaret \& Carroll, 2011). In some insects, AhR / ARNT may regulate the overexpression of multiple detoxification genes related to pesticide resistance (Hu et al., 2019). Transcription factor limpet has an impact on fungus-free insect survival, and these transcription factors have a direct effect on protecting Triatoma infestans from conditions of pathogenic pathogens, and these transcription factors are part of the primary immune response of other insects (Altincicek, Knorr \& Vilcinskas, 2008; Jin et al., 2008; Mannino, Paixão \& Pedrini, 2019). A total of 9,618 lncRNAs and 2,084 transcription factors of $R$. ferrugineus were obtained in our study. Furthermore, the discovery of these IncRNAs and TFs will provide certain reference information for further research on the function of $R$. ferrugineus in the growth, immunity and insecticide resistance.

\section{Conclusions}

PacBio Iso-Seq and Illumina RNA-seq were combined to successfully perform a full-length transcriptome of $R$. ferrugineus, and analyses of gene annotation, CDS prediction, transcription factors, SSR discovery, LncRNA prediction and alternative splicing were smoothly conducted without reference genome species. This research provides a valuable set of complete full-length 
400 transcripts for genomic reference, supplying an important and valuable basis for further study of 401 the growth and development of $R$. ferrugineus, as well as other congeneric insects.

\section{Acknowledgements}

403 We thank Professor Wei Yang for technical support (Provincial Key Laboratory of Forest

404 Protection, College of Forestry, Sichuan Agricultural University, Yaan City, China). We thank

405 Professor Wei Yan for samples support (Coconut Research Institute, Chinese Academy of

406 Tropical Agricultural Sciences, Wenchang City, China.). We also wish to express our deep

407 appreciation to the anonymous reviewers for their comments that helped to improve the quality

408 of this manuscript.

409 Abbreviations

$\begin{array}{ll}\text { FL } & \text { full-length } \\ \text { SMRT } & \text { single-molecule real-time } \\ \text { AS } & \text { alternative splicing } \\ \text { TFs } & \text { transcription factors } \\ \text { SSR } & \text { simple sequence repeats } \\ \text { lncRNAs } & \text { long noncoding RNAs } \\ \text { CDS } & \text { coding sequence } \\ \text { CCSs } & \text { circular consensus sequences } \\ \text { nFL } & \text { nonfull-length } \\ \text { FLNC } & \text { full-length non chimera } \\ \text { NR } & \text { Non-Redundant Protein Database } \\ \text { Swiss-Prot } & \text { A manually annotated and reviewed protein sequence database } \\ \text { Nt } & \text { NCBI non-redundant nucleotide sequences } \\ \text { Pfam } & \text { Protein family } \\ \text { GO } & \text { Gene Ontology } \\ \text { KOG } & \text { euKaryotic Ortholog Groups } \\ \text { KEGG } & \text { Kyoto Encyclopedia of Genes and Genomes } \\ \text { CNCI } & \text { Coding-Non-Coding-Index } \\ \text { CPC } & \text { Coding Potential Calculator } \\ \text { F_Adult } & \text { female adult } \\ \text { M_Adult } & \text { male adult } \\ \text { larva } & \text { 7th instars larva }\end{array}$

410

411 References

412 Abdel-Ghany SE, Hamilton M, Jacobi JL, Ngam P, Devitt N, Schilkey F, Ben-Hur A, Reddy

413 ASN. 2016. A survey of the sorghum transcriptome using single-molecule long reads. Nature

414 Communications 7:11706 DOI: 10.1038/ncomms11706.

415 Ahmed W, Liu ZF. 2018. Long non-coding RNAs: novel players in regulation of immune

416 response upon herpesvirus infection. Frontiers in Immunology 9:761 DOI:

417 10.3389/fimmu.2018.00761.

418 Altincicek B, Knorr E, Vilcinskas A. 2008. Beetle immunity: identification of immune-inducible

419 genes from the model insect Tribolium castaneum. Developmental and Comparative

420 Immunology 32:585-595 DOI: 10.1016/j.dci.2007.09.005. 
421 Altschul SF, Madden TL, Schaffer AA, Zhang J, Zhang Z, Miller W, Lipman DJ. 1997. Gapped 422 BLAST and PSI-BLAST: a new generation of protein database search programs. Nucleic Acids 423 Research 25:3389-3402 DOI: 10.1093/nar/25.17.3389.

424 Apweiler R, Bairoch A, Wu CH, Barker WC, Boeckmann B, Ferro S, Gasteiger E, Huang H, 425 Lopez R, Magrane M, Martin MJ, Natale DA, O'Donovan C, Redaschi N, Yeh LL. 2004.

426 UniProt: the universal protein knowledgebase. Nucleic Acids Research 32:D115-D119 DOI: 427 10.1093/nar/gkh131.

428 Ashburner M, Ball CA, Blake JA, Botstein D, Butler H, Cherry JM, Davis AP, Dolinski K, 429 Dwight SS, Eppig JT, Harris MA, Hill DP, Issel-Tarver L, Kasarskis A, Lewis S, Matese JC, 430 Richardson JE, Ringwald M, Rubin GM, Sherlock G. 2000. Gene Ontology: tool for the 431 unification of biology. Nature Genetics 25:25-29 DOI: 10.1016/S0315-5463(89)70537-X.

432 Au KF, Sebastiano V, Afshar PT, Durruthy JD, Lee L, Williams BA, van Bakel H, Schadt EE, 433 Reijo-Pera RA, Underwood JG, Wong WH. 2013. Characterization of the human ESC 434 transcriptome by hybrid sequencing. Proceedings of the National Academy of Sciences of the 435 United States of America 110:E4821-E4830 DOI: 10.1073/pnas.1320101110.

436 Batista PJ, Chang HY. 2013. Long noncoding RNAs: cellular address codes in development and 437 disease. Cell 152:1298-1307 DOI: 10.1016/j.cell.2013.02.012.

438 Blumberg D. 2008. Date palm arthropod pests and their management in Israel. Phytoparasitica 439 36:411-448.

440 Boyle MJ, Seaver EC. 2010. Expression of FOXA and GATA transcription factors correlates with 441 regionalized gut development in two lophotrochozoan marine worms: Chaetopterus (Annelida) 442 and Themiste lageniformis (Sipuncula). EvoDevo 1:1-18 DOI: 10.1186/2041-9139-1-2.

443 Braun S, Enculescu M, Setty ST, Cortes-Lopez M, de Almeida BP, Sutandy FXR, Schulz L, 444 Busch A, Seiler M, Ebersberger S, Barbosa-Morais NL, Legewie S, Koenig J, Zarnack K. 2018. 445 Decoding a cancer-relevant splicing decision in the RON proto-oncogene using high-throughput 446 mutagenesis. Nature Communications 9:3315 DOI: 10.1038/s41467-018-05748-7.

447 Chanchay P, Vongsangnak W, Thancharoen A, Sriboonlert A. 2019. Reconstruction of insect 448 hormone pathways in an aquatic firefly, Sclerotia aquatilis (Coleoptera: Lampyridae), using 449 RNA-seq. PeerJ 7:e7428 DOI: 10.7717/peerj.7428.

450 Chen K, Rajewsky N. 2007. The evolution of gene regulation by transcription factors and 451 microRNAs. Nature Reviews Genetics 8:93-103 DOI: 10.1038/nrg1990.

452 Chen Y, Dai H, Chen S, Zhang L, Long M. 2011. Highly Tissue Specific Expression of Sphinx 453 Supports Its Male Courtship Related Role in Drosophilamelanogaster. PLOS ONE 6:e18853 454 DOI: 10.1371/journal.pone.0018853.

455 Chen SY, Deng F, Jia X, Li C, Lai SJ. 2017. A transcriptome atlas of rabbit revealed by PacBio 456 single-molecule long-read sequencing. Scientific Reports 7: 7648 DOI: 10.1038/s41598-017457 08138-z.

458 Coghlan A, Fiedler TJ, McKay SJ, Flicek P, Harris TW, Blasiar D, Stein LD, Consortium TN. 459 2008. nGASP--the nematode genome annotation assessment project. BMC Bioinformatics 9:549 460 DOI: $10.1186 / 1471-2105-9-549$. 
461 Deng YY, Li JQ, Wu SF, Zhu YP, Chen YW, He FC, Chen YW, Deng Y, Li J, Wu S, Zhu Y, 462 Chen Y, He F, Deng L, LI J, Wu S, Zhu Y, Chen Y, He F, Deng Y, Li J, Wu S, Zhu Y, Deng Y, 463 Li J, Wu S, He F, Wu SF, Zhu Y, Chen Y, Wu S, He F, Deng M, Li JT, Zhu Y, He FY. 2006. 464 Integrated nr Database in Protein Annotation System and Its Localization. Computer 465 Engineering 32:71-73 DOI: 10.1109/INFOCOM.2006.241.

466 Djebali S, Davis CA, Merkel A, Dobin A, Lassmann T, Mortazavi A, Tanzer A, Lagarde J, Lin 467 W, Schlesinger F, Xue C, Marinov GK, Khatun J, Williams BA, Zaleski C, Rozowsky J, Der 468 MR, Kokocinski F, Abdelhamid RF, Alioto T, Antoshechkin I, Baer MT, Bar NS, Batut P, Bell 469 K, Bell I, Chakrabortty S, Chen X, Chrast J, Curado J, Derrien T, Drenkow J, Dumais E, Dumais 470 J, Duttagupta R, Falconnet E, Fastuca M, Fejes-Toth K, Ferreira P, Foissac S, Fullwood MJ, Gao 471 H, Gonzalez D, Gordon A, Gunawardena H, Howald C, Jha S, Johnson R, Kapranov P, King B, 472 Kingswood C, Luo OJ, Park E, Persaud K, Preall JB, Ribeca P, Risk B, Robyr D, Sammeth M, 473 Schaffer L, See L, Shahab A, Skancke J, Suzuki AM, Takahashi H, Tilgner H, Trout D, Walters 474 N, Wang H, Wrobel J, Yu Y, Ruan X, Hayashizaki Y, Harrow J, Gerstein M, Hubbard T, 475 Reymond A, Antonarakis SE, Hannon G, Giddings MC, Ruan Y, Wold B, Carninci P, Guigo R, 476 Gingeras TR. 2012. Landscape of transcription in human cells. Nature 489:101-108 DOI:

$477 \quad 10.1038$ /nature11233.

478 Ekblom R, Galindo J. 2011. Applications of next generation sequencing in molecular ecology of 479 non-model organisms. Heredity 107:1-15 DOI: 10.1038/hdy.2010.152.

480 Etebari K, Furlong MJ, Asgari S. 2015. Genome wide discovery of long intergenic non-coding 481 RNAs in Diamondback moth (Plutella xylostella) and their expression in insecticide resistant 482 strains. Scientific Reports 5:14642 DOI: 10.1038/srep14642.

483 Ferry M, Gomez S. 2002. The red palm weevil in the Mediterranean area. Palms 46:172-178. 484 Finn RD, Bateman A, Clements J, Coggill P, Eberhardt RY, Eddy SR, Heger A, Hetherington K, 485 Holm L, Mistry J, Sonnhammer ELL, Tate J, Punta M. 2014. Pfam: the protein families 486 database. Nucleic Acids Research 42:D222-D230 DOI: 10.1093/nar/gkt1223.

487 Finn RD, Coggill P, Eberhardt RY, Eddy SR, Mistry J, Mitchell AL, Potter SC, Punta M, 488 Qureshi M, Sangrador-Vegas A, Salazar GA, Tate J, Bateman A. 2016. The Pfam protein 489 families database: towards a more sustainable future. Nucleic Acids Research 44:D279-D285 490 DOI: $10.1093 /$ nar/gkv1344.

491 Fitzgerald KA, Caffrey DR. 2014. Long noncoding RNAs in innate and adaptive immunity. 492 Current Opinion in Immunology 26:140-146 DOI: 10.1016/j.coi.2013.12.001.

493 Fu L, Niu B, Zhu Z, Wu S, Li W. 2012. CD-HIT: accelerated for clustering the next-generation 494 sequencing data. Bioinformatics 28:3150-3152 DOI: 10.1093/bioinformatics/bts565.

495 Ge X, He S, Wang T, Yan W, Zong S. 2015. Potential Distribution Predicted for Rhynchophorus 496 ferrugineus in China under Different Climate Warming Scenarios. PLOS ONE 10(10): e0141111 497 DOI: 10.1371/journal.pone.0141111.

498 Habineza P, Muhammad A, Ji T, Xiao R, Yin X, Hou Y, and Shi Z. 2019. The Promoting Effect 499 of Gut Microbiota on Growth and Development of Red Palm Weevil, Rhynchophorus 500 ferrugineus (Olivier) (Coleoptera: Dryophthoridae) by Modulating Its Nutritional Metabolism. 
501 Frontiers in Microbiology 10:1212 DOI: 10.3389/fmicb.2019.01212.

502 Hartley SW, Mullikin JC, Klein DC, Park M, Coon SL. 2016. Alternative Isoform Analysis of

503 Ttc8 Expression in the Rat Pineal Gland Using a Multi-Platform Sequencing Approach Reveals

504 Neural Regulation. PLOS ONE 11:e163590 DOI: 10.1371/journal.pone.0163590.

505 Heward JA, Lindsay MA. 2014. Long non-coding RNAs in the regulation of the immune

506 response. Trends in Immunology 35:408-419 DOI: 10.1016/j.it.2014.07.005.

507 Hittinger, CT, Johnston M, Tossberg JT, and Rokas A. 2010. Leveraging skewed transcript

508 abundance by RNA-Seq to increase the genomic depth of the tree of life. Proceedings of the

509 National Academy of Sciences 107:1476-1481 DOI: 10.1073/pnas.0910449107.

$510 \mathrm{Hu}$ B, Huang H, Wei Q, Ren M, Mburu DK, Tian X, Su J. 2019. Transcription factors CncC/Maf 511 and AhR/ARNT coordinately regulate the expression of multiple GSTs conferring resistance to

512 chlorpyrifos and cypermethrin in Spodoptera exigua. Pest Management Science 75:2009-2019

513 DOI: $10.1002 /$ ps.5316.

514 Hussain A, Rizwan-ul-Haq M, Al-Jabr AM, Al-Ayied HY. 2013. Managing invasive populations

515 of red palm weevil: A worldwide perspective. Journal of Food Agriculture and Environment

516 11:456-463 DOI: 10.1146/annurev-food-022811-101247.

517 Jia D, Wang Y, Liu Y, Hu J, Guo Y, Gao L, and Ma R. 2018. SMRT sequencing of full-length

518 transcriptome of flea beetle Agasicles hygrophila (Selman and Vogt). Scientific Reports 8:2197

519 DOI: 10.1038/s41598-018-20181-y.

520 Jin LH, Shim J, Yoon JS, Kim B, Kim J, Kim-Ha J, Kim Y. 2008. Identification and functional

521 analysis of antifungal immune response genes in Drosophila. PLoS Pathogens 4(10):e1000168

522 DOI: 10.1371/journal.ppat.1000168.

523 Kanehisa M, Goto S, Kawashima S, Okuno Y, Hattori M. 2004. The KEGG resource for

524 deciphering the genome. Nucleic Acids Research 32:D277-D280 DOI: 10.1093/nar/gkh063.

525 Kawamoto M, Jouraku A, Toyoda A, Yokoi K, Minakuchi Y, Katsuma S, Fujiyama A, Kiuchi T,

526 Yamamoto K, Shimada T. 2019. High-quality genome assembly of the silkworm, Bombyx mori.

527 Insect Biochemistry and Molecular Biology 107:53-62 DOI: 10.1016/j.ibmb.2019.02.002.

528 Kong L, Zhang Y, Ye Z, Liu X, Zhao S, Wei L, Gao G. 2007. CPC: assess the protein-coding

529 potential of transcripts using sequence features and support vector machine. Nucleic Acids

530 Research 35:W345-W349 DOI: 10.1093/nar/gkm391.

531 Koonin EV, Fedorova ND, Jackson JD, Jacobs AR, Krylov DM, Makarova KS, Mazumder R, 532 Mekhedov SL, Nikolskaya AN, Rao BS, Rogozin IB, Smirnov S, Sorokin AV, Sverdlov AV,

533 Vasudevan S, Wolf YI, Yin JJ, Natale DA. 2004. A comprehensive evolutionary classification of

534 proteins encoded in complete eukaryotic genomes. Genome Biology 5:R7 DOI: 10.1186/gb-

535 2004-5-2-r7.

536 Koren S, Schatz MC, Walenz BP, Martin J, Howard JT, Ganapathy G, Wang Z, Rasko DA, 537 McCombie WR, Jarvis ED, Phillippy AM. 2012. Hybrid error correction and de novo assembly 538 of single-molecule sequencing reads. Nature Biotechnology 30:693-700 DOI: 10.1038/nbt.2280.

539 Kung JTY, Colognori D, Lee JT. 2013. Long Noncoding RNAs: Past, Present, and Future.

540 Genetics 193:651-669 DOI: 10.1534/genetics.112.146704. 
541 Lakhotia SC. 2012. Long non-coding RNAs coordinate cellular responses to stress. Wiley

542 Interdisciplinary Reviews: RNA 193:651-669 DOI: 10.1002/wrna.1134.

543 Larsen PA, Campbell CR, Yoder AD. 2014. Next-generation approaches to advancing eco-

544 immunogenomic research in critically endangered primates. Molecular Ecology Resources

545 14:1198-1209 DOI: 10.1111/1755-0998.12274.

546 Li M, Wen S, Guo X, Bai B, Gong Z, Liu X, Wang Y, Zhou Y, Chen X, Liu L, Chen R. 2012.

547 The novel long non-coding RNA CRG regulates Drosophila locomotor behavior. Nucleic Acids

548 Research 40:11714-11727 DOI: 10.1093/nar/gks943.

549 Li A, Zhang J, Zhou Z. 2014. PLEK: a tool for predicting long non-coding RNAs and messenger

550 RNAs based on an improved $k$-mer scheme. BMC Bioinformatics 15 DOI: 10.1186/1471-2105-

$551 \quad 15-311$.

552 Li Q, Li Y, Song J, Xu H, Xu J, Zhu Y, Li X, Gao H, Dong L, Qian J, Sun C, Chen S. 2014.

553 High-accuracy de novo assembly and SNP detection of chloroplast genomes using a SMRT

554 circular consensus sequencing strategy. New Phytologist 204:1041-1049 DOI:

$55510.1111 / \mathrm{nph} .12966$.

556 Li Y, Fang C, Fu Y, Hu A, Li C, Zou C, Li X, Zhao S, Zhang C, Li C. 2018. A survey of

557 transcriptome complexity in Sus scrofa using single-molecule long-read sequencing. DNA

558 Research 25:421-437 DOI: 10.1093/dnares/dsy014.

559 Li X, Wang H, Zhang Y, Zhang J, Qi S, Zhang Y, Gao M. 2019. Overexpression of lncRNA H19

560 changes basic characteristics and affects immune response of bovine mammary epithelial cells.

561 PeerJ 7:e6715 DOI: 10.7717/peerj.6715.

562 Lin H, Lin X, Zhu J, Yu X, Xia X, Yao F, Yang G, You M. 2017. Characterization and

563 expression profiling of serine protease inhibitors in the diamondback moth, Plutella xylostella

564 (Lepidoptera: Plutellidae). BMC Genomics 18 DOI: 10.1186/s12864-017-3583-z.

565 Mannino MC, Paixão FR, Pedrini N. 2019. The limpet transcription factors of Triatoma infestans

566 regulate the response to fungal infection and modulate the expression pattern of defensin genes.

567 Insect Biochemistry and Molecular Biology 108:53-60 DOI: 10.1016/j.ibmb.2019.03.010.

568 Mazza G, Francardi V, Simoni S, Benvenuti C, Cervo R, Faleiro JR, Llacer E, Longo S, Nannelli

569 R, Tarasco E, Roversi PF. 2014. An overview on the natural enemies of Rhynchophorus palm

570 weevils, with focus on R. ferrugineus. Biological Control 77:83-92 DOI:

571 10.1016/j.biocontrol.2014.06.010.

572 Mizutani R, Wakamatsu A, Tanaka N, Yoshida H, Tochigi N, Suzuki Y, Oonishi T, Tani H,

573 Tano K, Ijiri K, Isogai T, Akimitsu N. 2012. Identification and Characterization of Novel

574 Genotoxic Stress-Inducible Nuclear Long Noncoding RNAs in Mammalian Cells. PLOS ONE

575 7:e34949 DOI: 10.1371/journal.pone.0034949.

576 Muhammad A, Habineza P, Ji T, Hou Y, and Shi Z. 2019. Intestinal Microbiota Confer

577 Protection by Priming the Immune System of Red Palm Weevil Rhynchophorus ferrugineus

578 Olivier (Coleoptera: Dryophthoridae). Frontiers in Physiology 10:1303 DOI:

579 10.3389/fphys.2019.01303.

580 Mulvey BB, Olcese U, Cabrera JR, Horabin JI. 2014. An interactive network of long non-coding 
581 RNAs facilitates the Drosophila sex determination decision. Biochimica et Biophysica Acta 582 (BBA) - Gene Regulatory Mechanisms 1839:773-784 DOI: 10.1016/j.bbagrm.2014.06.007.

583 Nagalakshmi U, Wang Z, Waern K, Shou C, Raha D, Gerstein M, Snyder M. 2008. The 584 transcriptional landscape of the yeast genome defined by RNA sequencing. Science 320:13445851349 DOI: $10.1126 /$ science.1158441.

586 Noriega DD, Arias PL, Barbosa HR, Arraes FBM, Ossa GA, Villegas B, Coelho RR, 587 Albuquerque EVS, Togawa RC, Grynberg P, Wang H, Vélez AM, Arboleda JW, Grossi-de-Sa 588 MF, Silva MCM, Valencia-Jiménez A. 2019. Transcriptome and gene expression analysis of 589 three developmental stages of the coffee berry borer, Hypothenemus hampei. Scientific Reports 590 9:12804-12813 DOI: 10.1038/s41598-019-49178-x.

591 Qureshi IA, Mehler MF. 2013. Long Non-coding RNAs: Novel Targets for Nervous System

592 Disease Diagnosis and Therapy. Neurotherapeutics 10:632-646 DOI: 10.1007/s13311-013-01995930.

594 Sacchetti P. 2006. Identificazione, biologia e diffusione delcurculionide delle palme, 595 Rhynchophorus ferrugineus (Olivier). Informatore Fitopatologico 35-40.

596 Salmela L, Rivals E. 2014. LoRDEC: accurate and efficient long read error correction.

597 Bioinformatics 30:3506-3514 DOI: 10.1093/bioinformatics/btu538.

598 Sharon D, Tilgner H, Grubert F, Snyder M. 2013. A single-molecule long-read survey of the 599 human transcriptome. Nature biotechnology 31:1009-1014 DOI: 10.1038/nbt.2705.

600 Shi ZH, Lin YT, Hou YM. 2014. Mother-derived trans-generational immune priming in the red 601 palm weevil, Rhynchophorus ferrugineus Olivier (Coleoptera, Dryophthoridae). Bulletin of 602 Entomological Research 104:742-750 DOI: 10.1017/S0007485314000583.

603 Shimizu K, Adachi J, Muraoka Y. 2006. ANGLE: a sequencing errors resistant program for 604 predicting protein coding regions in unfinished cDNA. Journal of Bioinformatics and 605 Computational Biology 4:649-664 DOI: 10.1142/S0219720006002260.

606 Smith ER, Allis CD, Lucchesi JC. 2001. Linking global histone acetylation to the transcription 607 enhancement of X-chromosomal genes in Drosophila males. Journal of Biological Chemistry 608 276:31483-31486 DOI: 10.1074/jbc.C100351200.

609 Soshnev AA, Ishimoto H, McAllister BF, Li X, Wehling MD, Kitamoto T, Geyer PK. 2011. A 610 conserved long noncoding RNA affects sleep behavior in Drosophila. Genetics 189:455-468 611 DOI: 10.1534/genetics.111.131706.

612 Soroker V, Blumberg D, Haberman A, Hamburger-Rishard M, Reneh S, Talebaev S, 613 Anshelevich L, Harari AR. 2005. Current status of red palm weevil infestation in date palm 614 plantations in Israel. Phytoparasitica 33:97-106 DOI: 10.1007/BF02980931.

615 Tatusov RL, Fedorova ND, Jackson JD, Jacobs AR, Kiryutin B, Koonin EV, Krylov DM, 616 Mazumder R, Mekhedov SL, Nikolskaya AN, Rao BS, Smirnov S, Sverdlov AV, Vasudevan S, 617 Wolf YI, Yin JJ, Natale DA. 2003. The COG database: an updated version includes eukaryotes. 618 BMC Bioinformatics 4:41 DOI: 10.1186/1471-2105-4-41. 
619 Thatcher SR, Danilevskaya ON, Meng X, Beatty M, Zastrow-Hayes G, Harris C, Van Allen B, 620 Habben J, Li B. 2016. Genome-Wide Analysis of Alternative Splicing during Development and 621 Drought Stress in Maize. Plant Physiology 170:586-599 DOI: 10.1104/pp.15.01267.

622 Tilgner H, Grubert F, Sharon D, Snyder MP. 2014. Defining a personal, allele-specific, and 623 single-molecule long-read transcriptome. Proceedings of the National Academy of Sciences of 624 the United States of America 111:9869-9874 DOI : 10.1073/pnas.1400447111.

625 Valluri S, Rupam G, Srividya S. 2017. Long Non-Coding RNAs Regulating Immunity in Insects. 626 Non-Coding RNA 3(1):14 DOI: 10.3390/ncrna3010014.

627 Vatanparast M, Hosseininaveh V, Ghadamyari M, Sajjadian SM. 2014. Plant Cell Wall

628 Degrading Enzymes, Pectinase and Cellulase, in the Digestive System of the Red Palm Weevil, 629 Rhynchophorus ferrugineus (Coleoptera: Curculionidae). Plant Protection Science 50:190-198 630 DOI: 10.17221/43/2013-PPS.

631 Vuong CK, Black DL, Zheng S. 2016. The neurogenetics of alternative splicing. Nature Reviews 632 Neuroscience 17:265-281 DOI: 10.1038/nrn.2016.27.

633 Wakil W, Faleiro JR, Miller TA. 2015. Sustainable Pest Management in Date Palm:

634 CurrentStatus and Emerging Challenges, Sustainability in Plant and Crop Protection. Springer 635 International Publishing Switzerland.

636 Wang B, Tseng E, Regulski M, Clark TA, Hon T, Jiao Y, Lu Z, Olson A, Stein JC, Ware D. 637 2016. Unveiling the complexity of the maize transcriptome by single-molecule long-read 638 sequencing. Nature Communications 7:11708 DOI: 10.1038/ncomms11708.

639 Weirather JL, Afshar PT, Clark TA, Tseng E, Powers LS, Underwood JG, Zabner J, Korlach J, 640 Wong WH, Au KF. 2015. Characterization of fusion genes and the significantly expressed fusion 641 isoforms in breast cancer by hybrid sequencing. Nucleic Acids Research 43:e116 DOI:

$64210.1093 /$ nar/gkv562.

643 Won HI, Schulze TT, Clement EJ, Watson GF, Watson SM, Warner RC, Ramler E, Witte EJ, 644 Schoenbeck MA, Rauter CM, Davis PH. 2018. De novo Assembly of the Burying Beetle 645 Nicrophorus orbicollis (Coleoptera: Silphidae) Transcriptome Across Developmental Stages 646 with Identification of Key Immune Transcripts. J Genomics 6:41-52 DOI: 10.7150/jgen.24228.

647 Yang H, Cai Y, Zhuo Z, Yang W, Yang C, Zhang J, Yang Y, Wang B, Guan F. 2018.

648 Transcriptome analysis in different developmental stages of Batocera horsfieldi (Coleoptera:

649 Cerambycidae) and comparison of candidate olfactory genes. PLOS ONE 13:e192730 DOI:

650 10.1371/journal.pone.0192730.

651 Zaret KS, Carroll JS. 2011. Pioneer transcription factors: establishing competence for gene

652 expression. Genes \& Development 25:2227-2241 DOI: 10.1101/gad.176826.111.

653 Zhang H, Liu T, Liu C, Song S, Zhang X, Liu W, Jia H, Xue Y, Guo A. 2015. AnimalTFDB 2.0:

654 a resource for expression, prediction and functional study of animal transcription factors. Nucleic 655 Acids Research 43:D76-D81 DOI: 10.1093/nar/gku887.

656 Zhao L. 2018. Analysis of full-length transcriptome and mitochondrial transcriptome of three 657 orthoptera insects. D. Phil. Thesis, Shaanxi Normal University. 
658 Zhu J, Jiang F, Wang X, Yang P, Bao Y, Zhao W, Wang W, Lu H, Wang Q, Cui N, Li J, Chen

659 X, Luo L, Yu J, Kang L, Cui F. 2017. Genome sequence of the small brown planthopper,

660 Laodelphax striatellus. GigaScience 6(12) DOI: 10.1093/gigascience/gix 109.

661 Zhu B, Liang P, Gao X. 2016. Long noncoding RNAs (lncRNAs) and their research advances in

662 entomology. Acta Entomologica Sinica 59:1272-1281 DOI: 10. 16380/j. kcxb. 2016. 11. 016.

663

664 
Figure 1

Length distribution of $R$. ferrugineus unigenes obtained by PacBio Iso-Seq.

\section{Unigene Length Distribution}

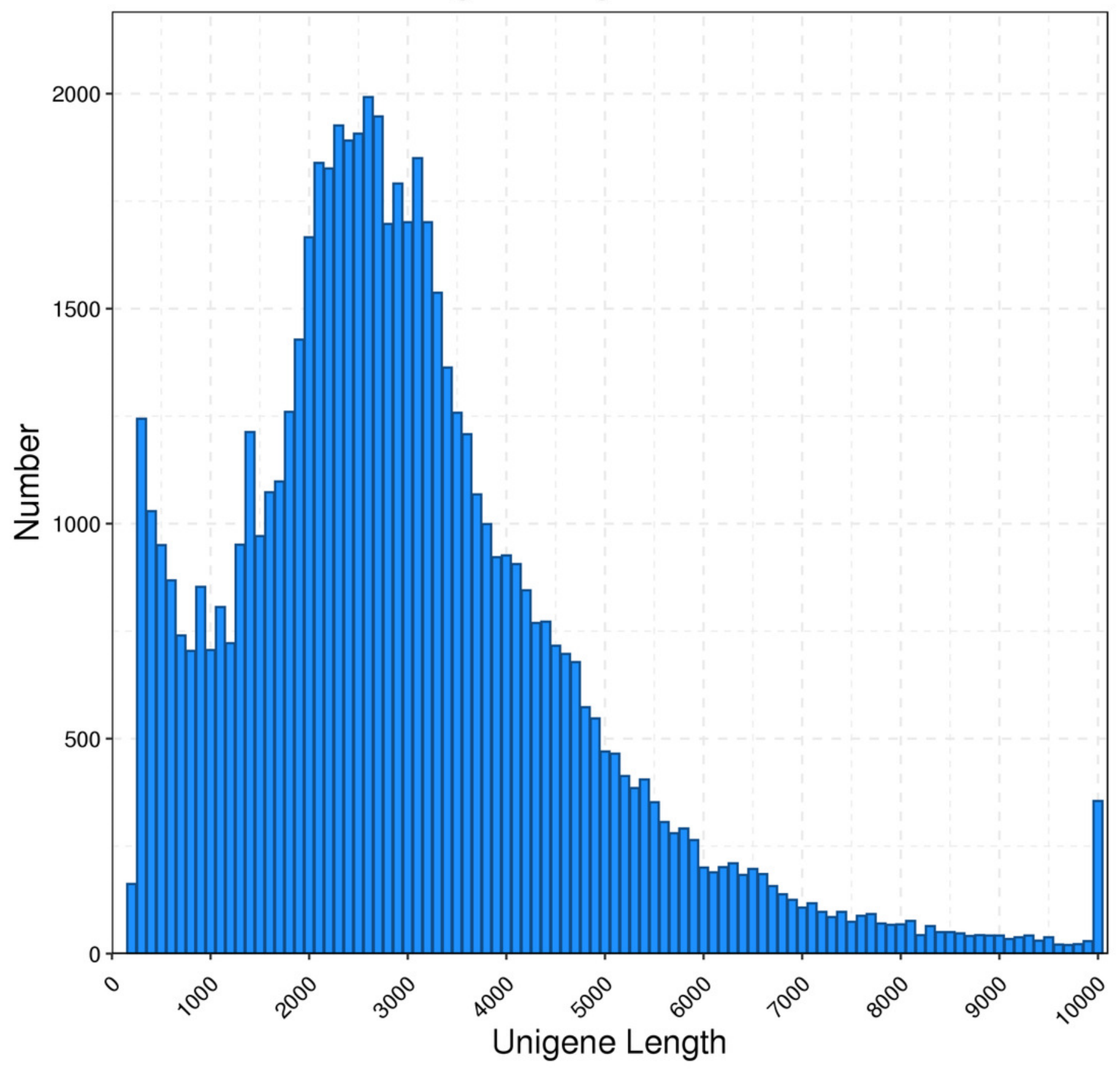




\section{Figure 2}

Function annotation of $R$. ferrugineus transcripts in all databases.

Note: NR, Non-Redundant Protein Database. Swiss-Prot, a manually annotated and reviewed protein sequence database. KEGG, Kyoto Encyclopedia of Genes and Genomes. KOG, euKaryotic Ortholog Groups. GO, Gene Ontology. NT, NCBI non-redundant nucleotide sequences. Pfam, Protein family.

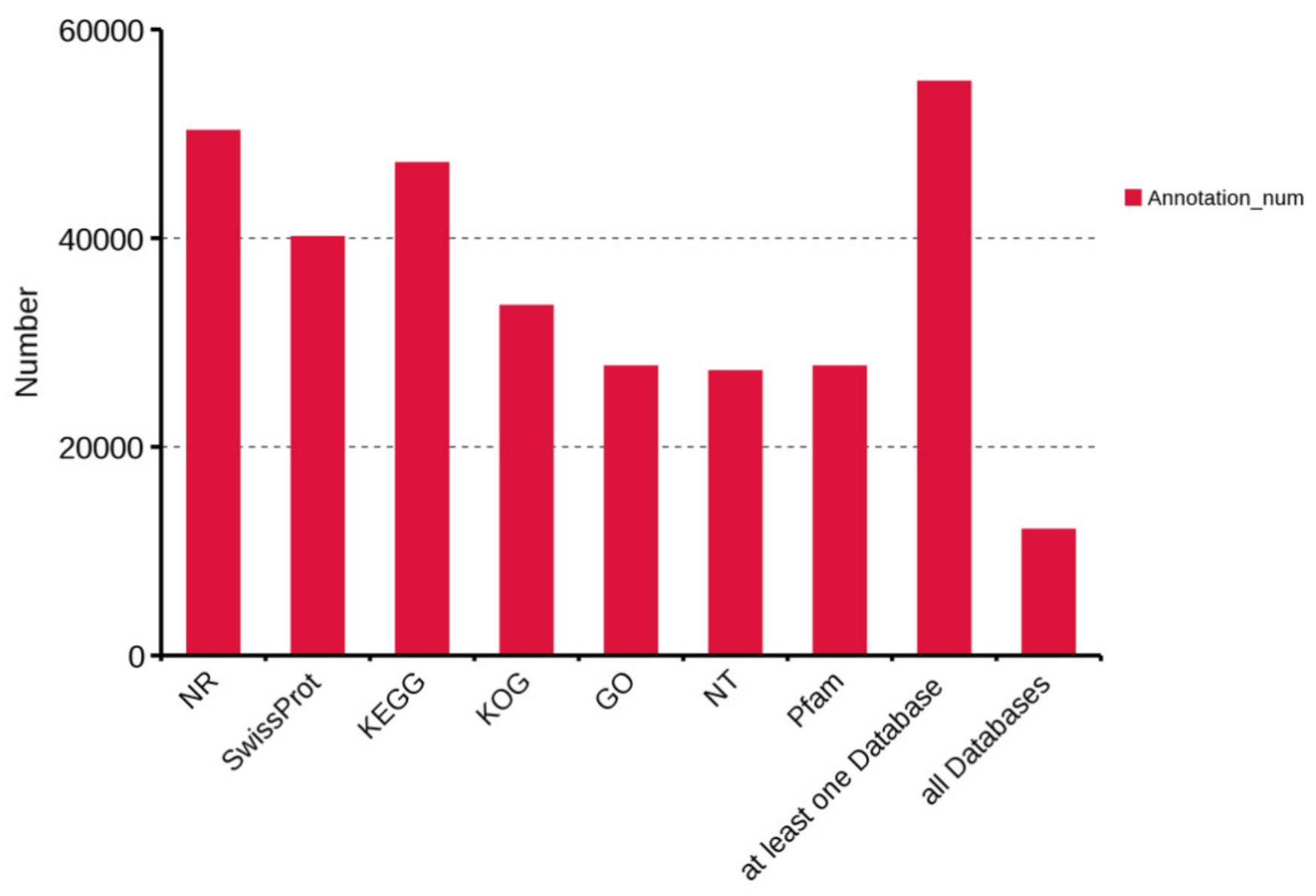

Database 
Figure 3

NR Homologous species distribution diagram of $R$. ferrugineus transcripts.

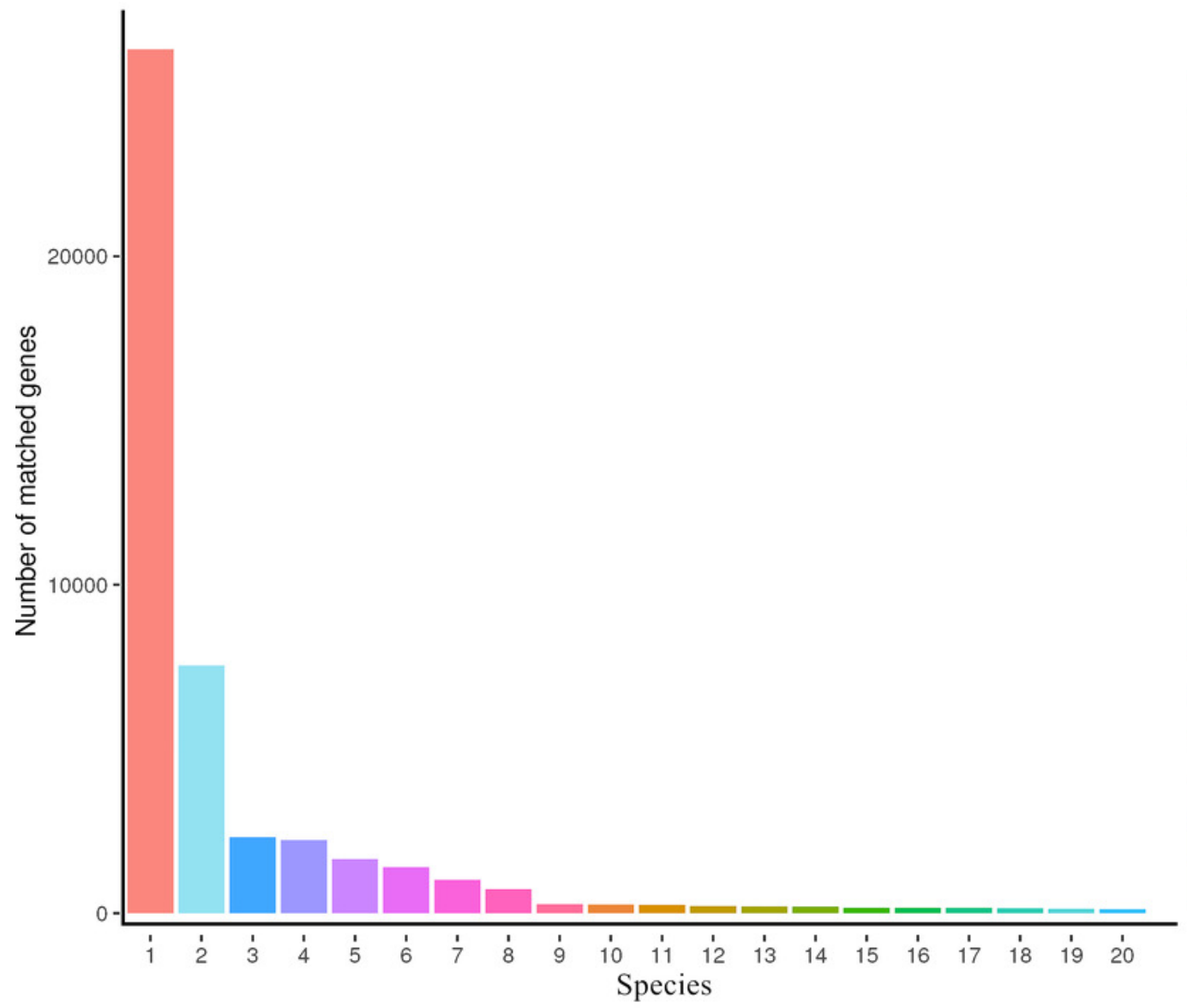
1 : Dendroctonus ponderosae
2 : Anoplophora glabripennis
3 : Bactrocera tryoni
4 : Tribolium castaneum
5 : Aethina tumida
6 : Lasius niger
7 : Clastoptera arizonana
8: Rhynchophorus ferrugineus
9 : Cyphomyrmex costatus
10 : Agrilus planipennis
11 : Lissorhoptrus oryzophilus
12 : Harpegnathos saltator
13 : Nicrophorus vespilloides
14 : Orussus abietinus
15 : Thermobia domestica
16 : Nyssomyia neivai
17 : Oryctes borbonicus
18 : Trachymyrmex septentrionalis
19 : Acromyrmex echinatior
20 : Acyrthosiphon pisum

Species 
Figure 4

KOG classification diagram of $R$. ferrugineus transcripts.

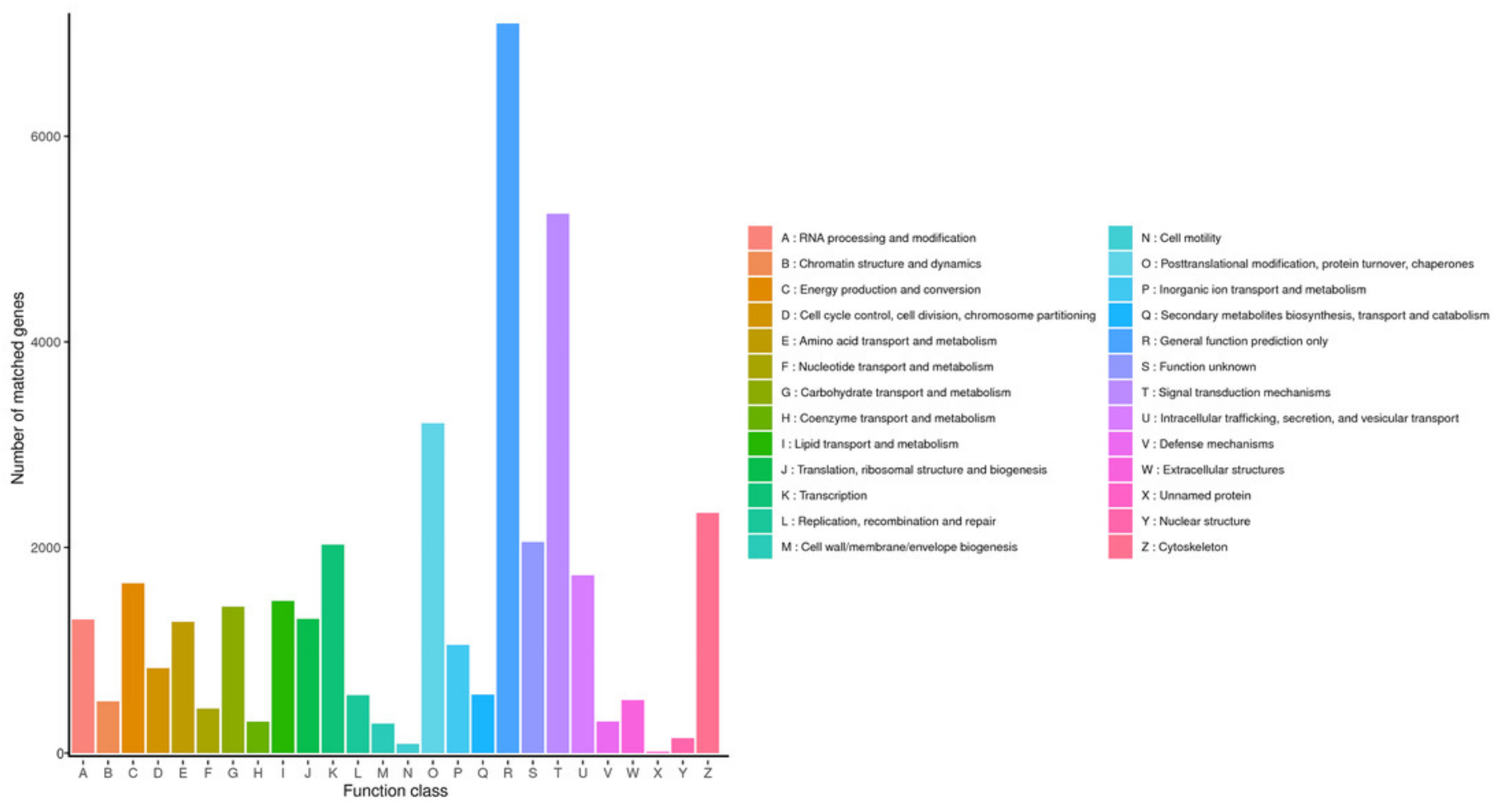


Figure 5

GO classification diagram of $R$. ferrugineus transcripts.

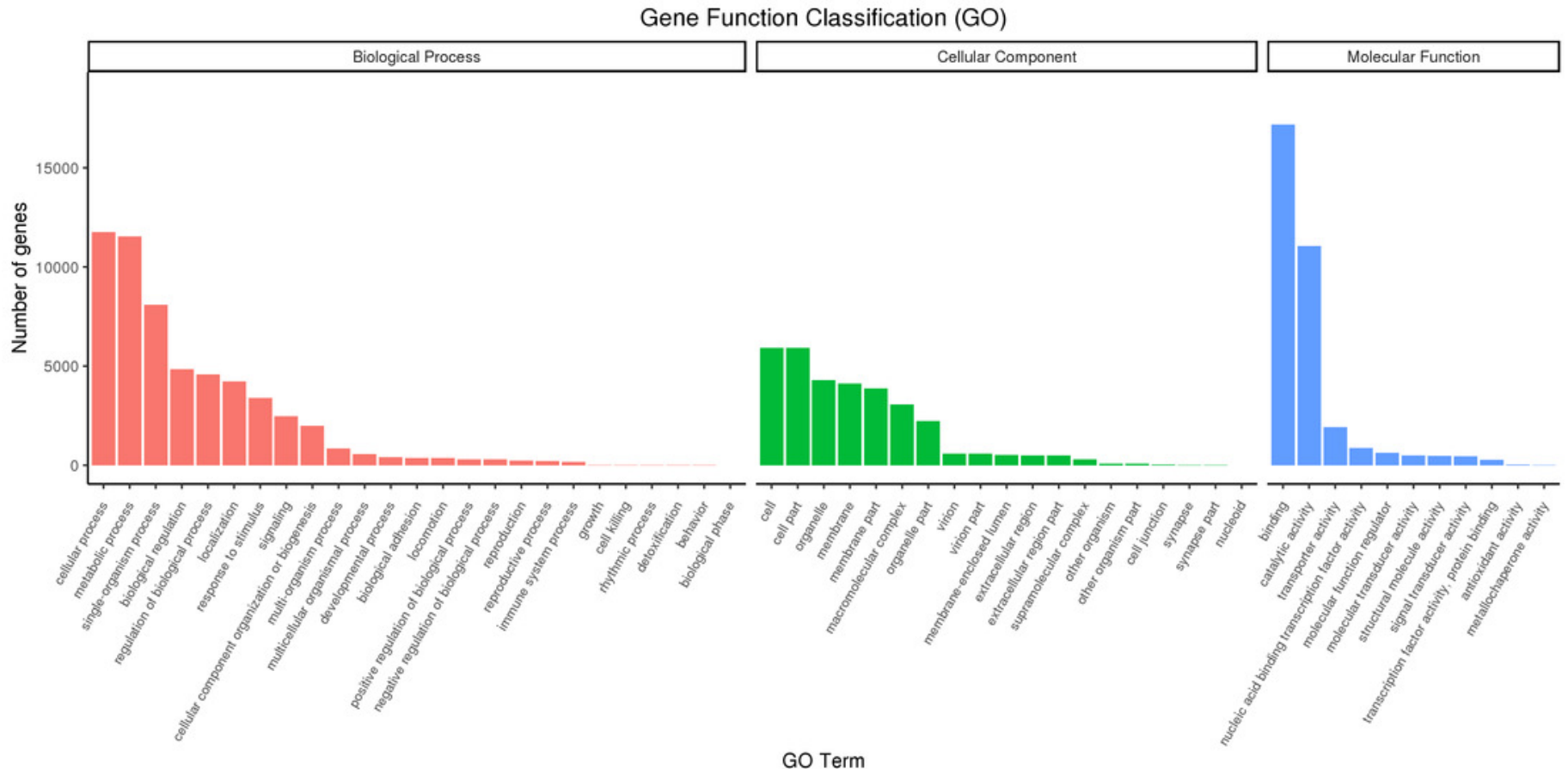




\section{Figure 6}

KEGG Pathway classification diagram of $R$. ferrugineus transcripts.

\section{KEGG pathway annotation}
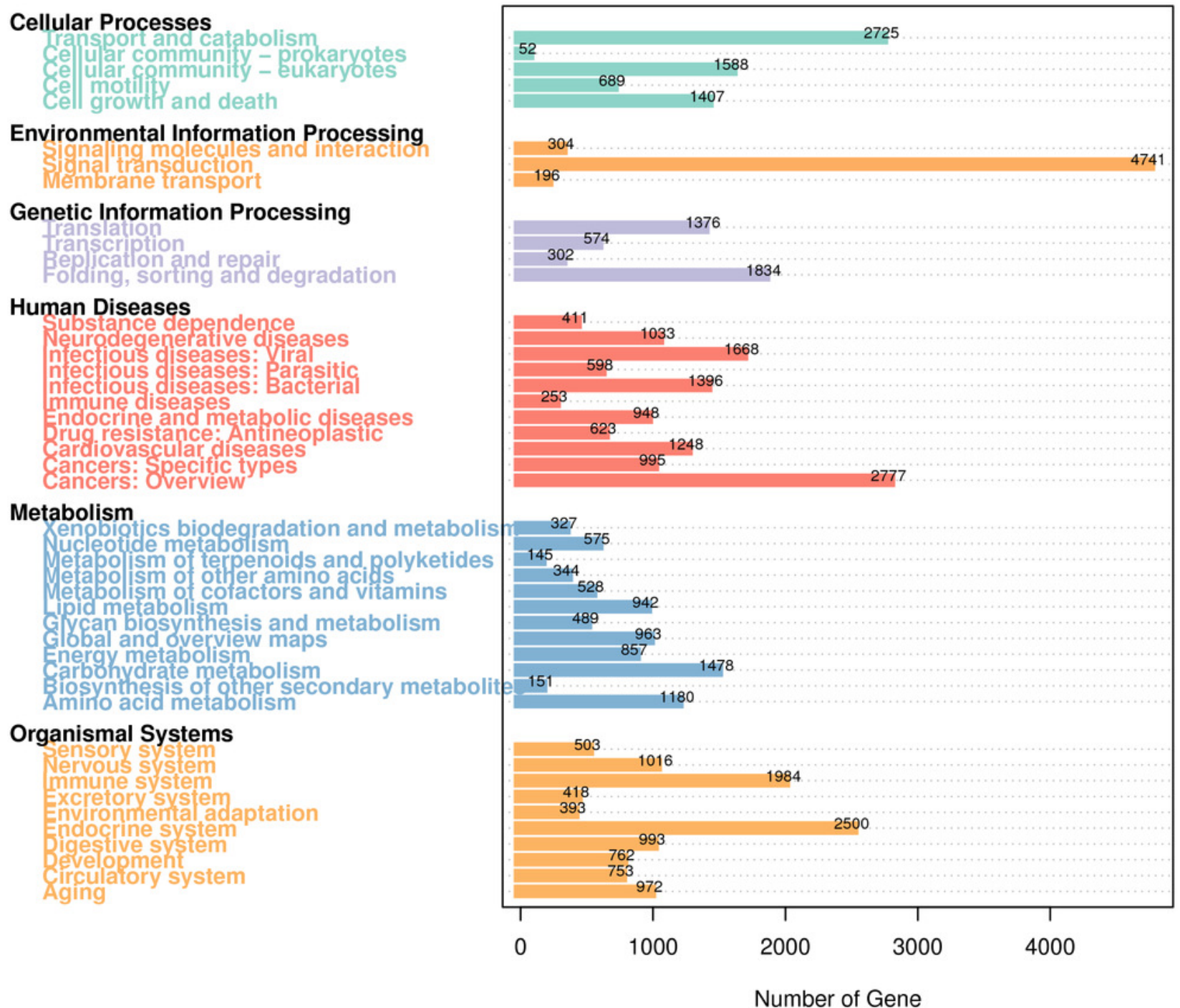
Figure 7

Number, percentage and length distributions of coding sequences of $R$. ferrugineus transcripts.

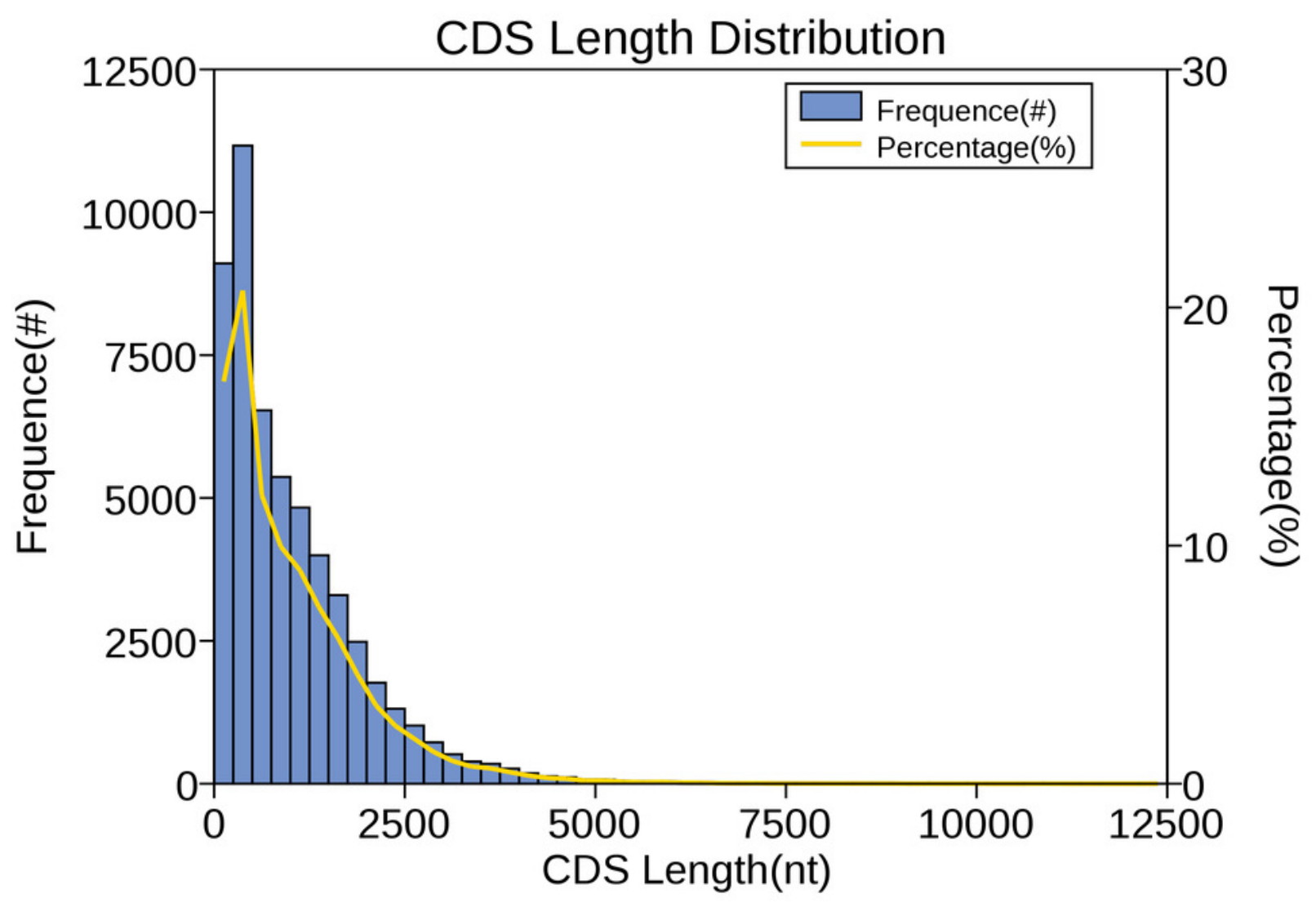


Figure 8

Number and family of top 29 transcription factors predicted by SMRT.

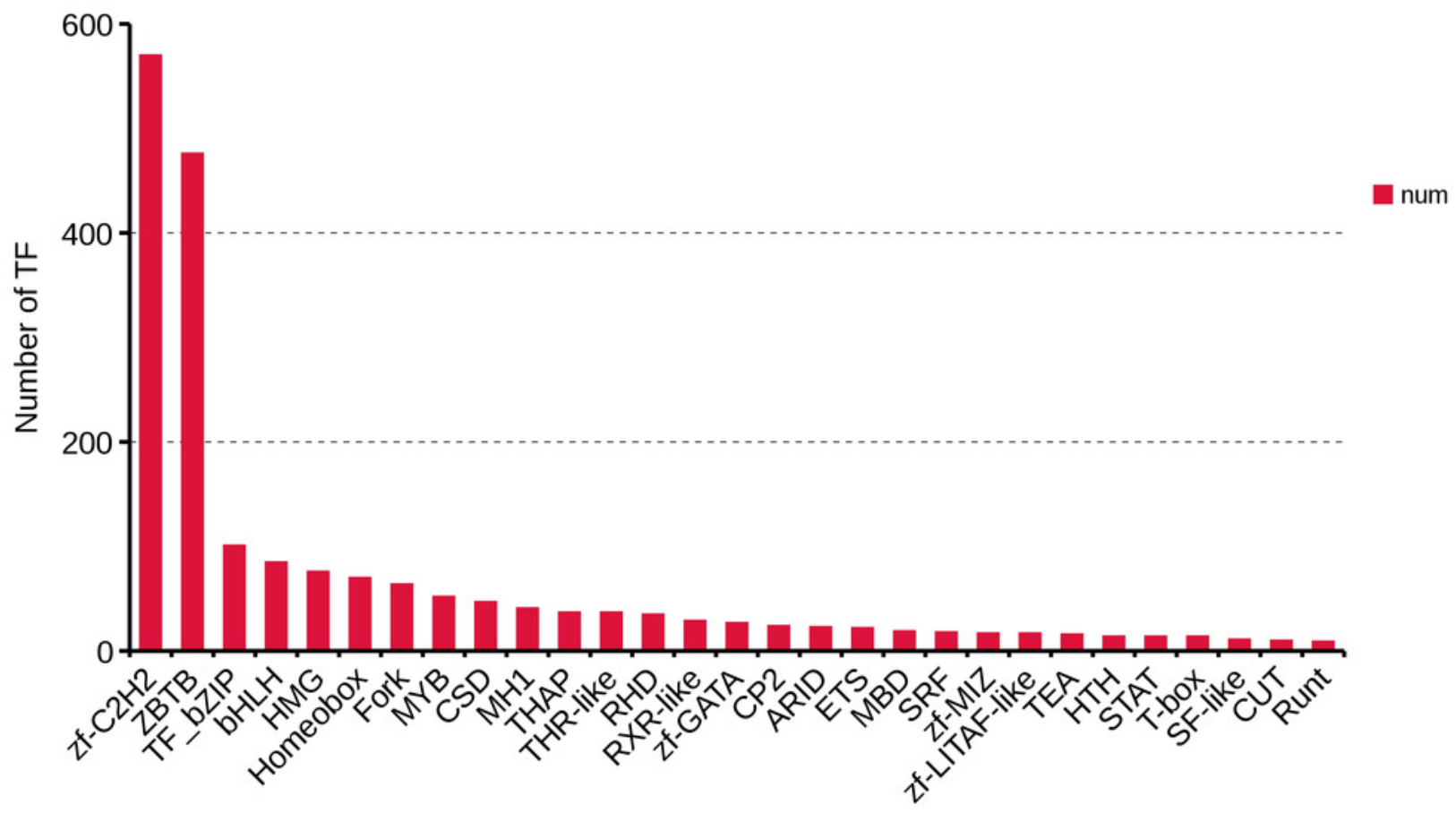

Transcript family 
Figure 9

Scattergram of simple sequence repeats of $R$. ferrugineus transcripts.

\section{Distribution of SSR Motifs}

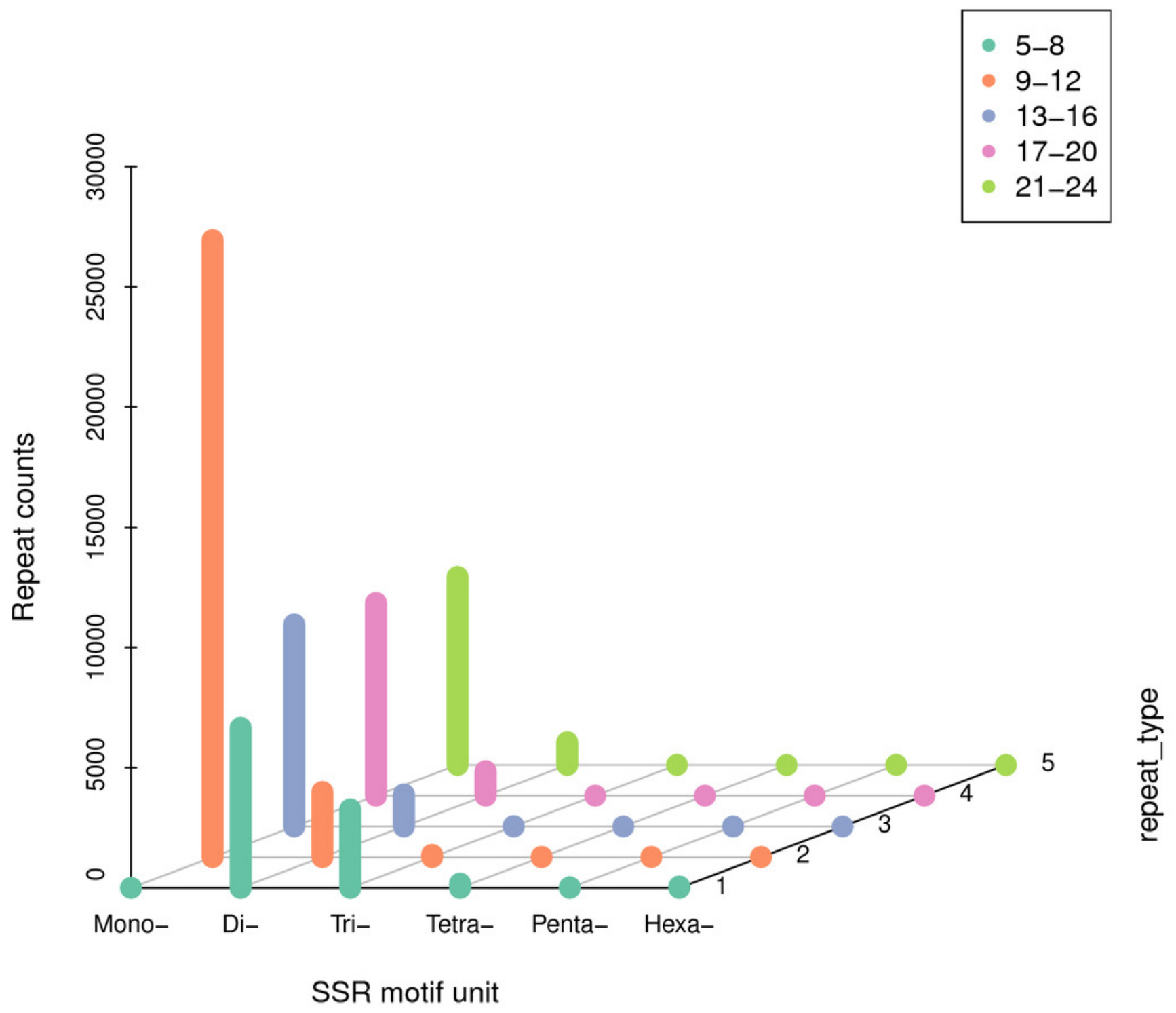




\section{Figure 10}

Venn diagram of IncRNA transcripts identified from PLEK, CNCl, CPC and Pfam.

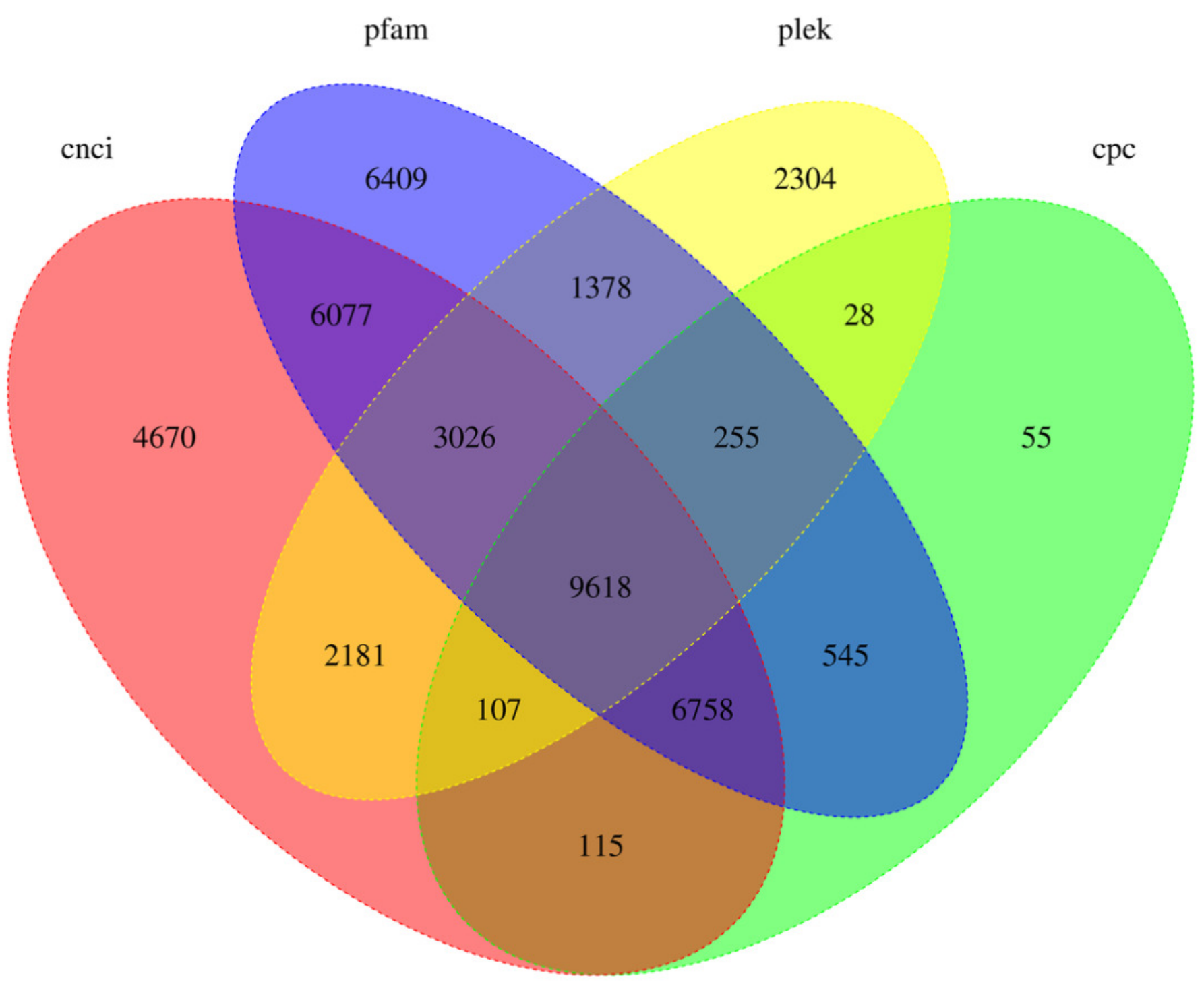


Figure 11

Length distribution of LncRNA and mRNA in $R$. ferrugineus.

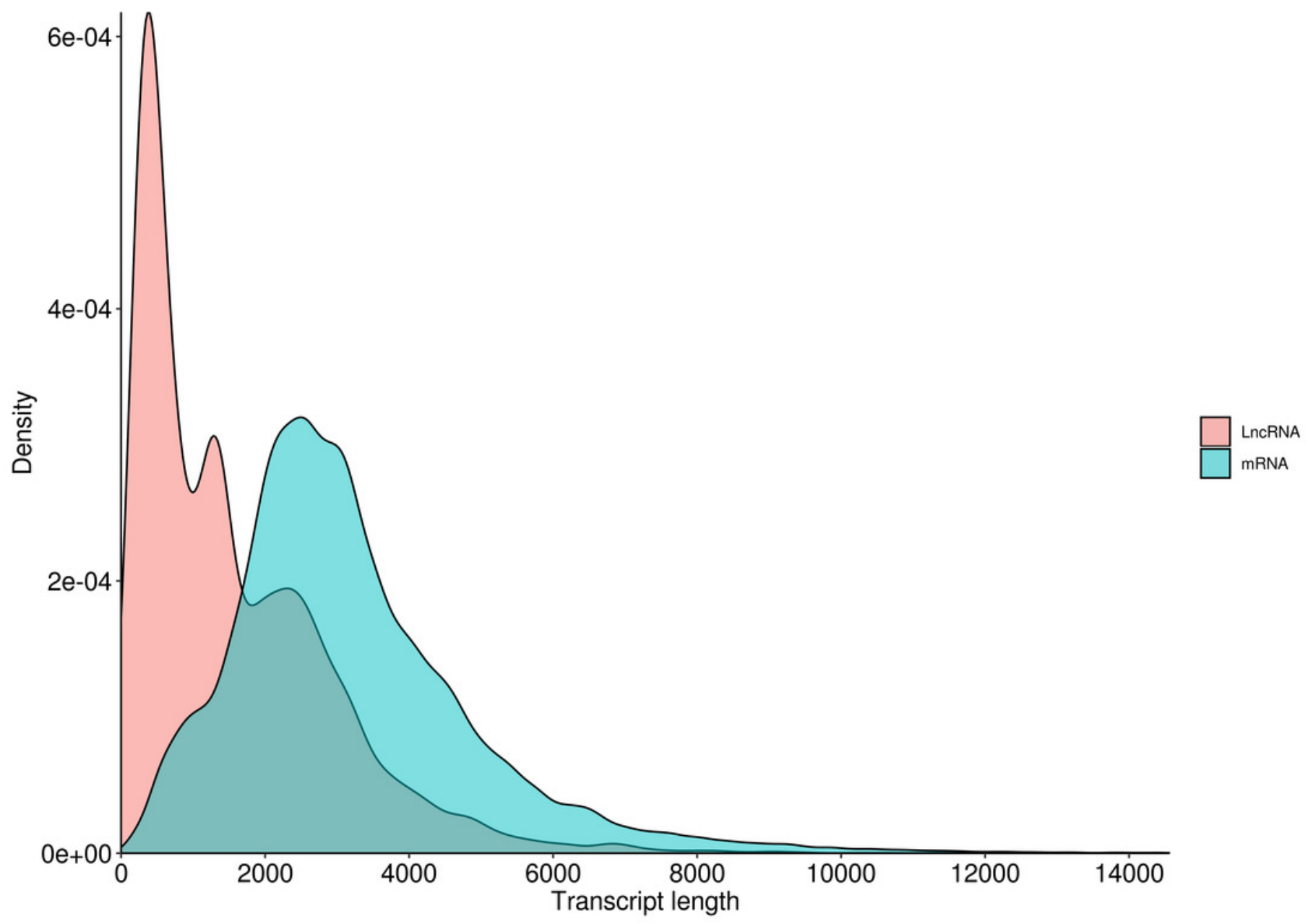

